\title{
BEST UNIFORM APPROXIMATION BY MEROMORPHIC FUNCTIONS WITH FREE POLES
}

\author{
S. J. POREDA
}

\begin{abstract}
Using some recent results on best polynomial approximations, a method for explicitly calculating the meromorphic function with a fixed number of free poles of best uniform approximation to certain functions on a closed Jordan curve is obtained.
\end{abstract}

For a complex valued function $f$ defined on a compact set $E$ in the plane, let $\|f\|_{E}=\max _{Z \in E}|f(z)|$. If $f$ is also continuous and nonvanishing on $\Gamma$, a closed Jordan curve, let $\Delta(f, \Gamma)$ denote the winding number of the closed curve $f(\Gamma)$ with respect to the origin.

Using some of this author's recent results [1], a method for calculating best meromorphic approximants with a fixed number of free poles to a certain class of functions on a closed Jordan curve is found. We begin by considering the special case where that curve is the unit circle $U$. The more general case will be discussed briefly later.

THEOREM 1. Let $f(z)=\sigma(z) / \prod_{k=1}^{n}\left(z-a_{k}\right)$ where the $a_{k}$ 's are distinct, $\left|a_{k}\right|<1$ for $k=1,2, \cdots, n$, and $\sigma(z)$ is regular in $D=\{|z|<1\}$ and continuous in $\bar{D}$. There then exists a unique function $r(z)$ meromorphic in $D$, continuous in $\bar{D}$, having $n-1$ or fewer poles in $D$ of best uniform approximation to $f$ on $U$. Furthermore, $r(z)$ is given by

$$
r(z)=f(z)-\lambda\left[\prod_{j=1}^{K}\left(\frac{1-\bar{c}_{j} z}{z-c_{j}}\right)\right]\left[\prod_{k=1}^{n}\left(\frac{1-\bar{a}_{k} z}{z-a_{k}}\right)\right],
$$

where $K \leqq n-1 ; \lambda$ is a prescribed root of an explicitly determined polynomial and the $c_{j}$ 's are in $D$ and can be calculated in terms of $\lambda$, the $a_{k}$ 's and the values $\sigma\left(a_{k}\right), k=1,2, \cdots, n$.

Proof. Let

$$
B_{k}=\sigma\left(a_{k}\right) / \prod_{j=1}^{n}\left(1-\bar{a}_{j} a_{k}\right), \text { for } k=1,2, \cdots, n .
$$

Received by the editors September 29, 1972 and, in revised form, May 31, 1973. AMS (MOS) subject classifications (1970). Primary 41A50; Secondary 41A20.

Key words and phrases. Best uniform approximation, closed Jordan curve. 
There exists a unique function $F(z)$ regular in $D$, continuous in $\bar{D}$ with values $F\left(a_{k}\right)=B_{k}^{-1}$ for $k=1,2, \cdots, n$ for which $\|F\|_{U}$ is a minimum. Furthermore [1], $F$ is of the form,

$$
F(z)=\frac{1}{\lambda} \prod_{j=1}^{K}\left(\frac{z-c_{j}}{1-\bar{c}_{j} z}\right),
$$

where $K, \lambda$ and the $c_{j}$ 's are as described above. Theorem 1 will follow from the following lemma.

LEMMA 1. Let $F(z)$ and the $B_{k}$ 's be as above. The function $R(z)=F(z)^{-1}$ is then the unique function that is meromorphic in $D$, continuous in $\bar{D}$ with $n-1$ or fewer poles in $D$ and values $R\left(a_{k}\right)=B_{k}$ for $k=1,2, \cdots, n$ for which $\|R\|_{U}$ is a minimum.

Furthermore, if $R^{*}(z)$ is meromorphic in $D$ and continuous in $\bar{D}$, with $n-M-1$ or fewer poles in $D$ and values $R^{*}\left(a_{k}\right)=B_{k}$ for $k=1,2, \cdots$, $n-M$, then $\left\|R^{*}\right\|_{U}>\|R\|_{U}$.

Proof. Suppose $R^{*}$ is as above and $\left\|R^{*}\right\|_{U} \leqq\|R\|_{U}$. Let us first consider the case where $\left\|R^{*}\right\|_{U}<\|R\|_{U}$. The function $R(z)-R^{*}(z)$ would then have at least $n-M$ zeros in $D$. Furthermore,

$$
\left\|R(z)-\left[R(z)-R^{*}(z)\right]\right\|<\|R(z)\|=\lambda \text { for all } z \in U ;
$$

and so it follows that

$$
\operatorname{Re} R(z)\left[\overline{R(z)-R^{*}(z)}\right]>0 \quad \text { for all } z \in U .
$$

This implies that as $z$ traverses $U,\left[R(z)-R^{*}(z)\right]$ lies in the half plane $|\arg w-\arg R(z)|<\pi / 2$ (where arg is a suitably chosen branch of the argument function for each $z$ ). Consequently the winding numbers of the image of $U$ under $R$ and $\left[R-R^{*}\right]$ must be equal. That is, $\Delta(R, U)=$ $\Delta\left(\left[R-R^{*}\right], U\right)$. However, $-\Delta(R, U)=\#$ of poles $R$ has in $D$, and so

$$
\begin{aligned}
\Delta\left(\left[R-R^{*}\right], U\right) & \geqq(n-M)+\Delta(R, U)-(n-M-1) \\
& =\Delta(R, U)+1,
\end{aligned}
$$

which thus yields a contradiction.

We may therefore assume that $\left\|R^{*}\right\|_{U}=\|R\|_{U}$. It then follows [2] that $\left|R^{*}(z)\right|=\|R\|_{U}=\lambda$ for all $z \in U$ since otherwise there exists a polynomial $p(z)$ such that

$$
\left\|R^{*}(z)-p(z) \prod_{k=1}^{n}\left(\frac{z-a_{k}}{1-\bar{a}_{k} z}\right)\right\|_{U} \leqq\left\|R^{*}\right\|_{U}
$$


and so we are again led to a contradiction. Furthermore, as a consequence of Rouché's theorem, $R^{*}$ must be a finite Blaschke product. Let $N$ denote the number of zeros and $J$ the number of poles that $R^{*}$ has in $D(J \leqq n-$ $M-1)$.

Now since both $R$ and $R^{*}$ are finite Blaschke products having the same uniform norm on $U$, it follows that $R(1 / \bar{z})=R^{*}(1 / \bar{z})$ whenever $R(z)=$ $R^{*}(z)$. Since $R(z)=R^{*}(z)$ has at least $n-M$ roots in $D$, it therefore has at least $n-M$ roots in $\{|z|>1\}$. Furthermore, it will have at least $|N+K-J|$ roots on $U$ and the total number of roots is no more than $K+N+J$.

If $N+K-J \geqq 0$, then we can write

$$
N+K+J \geqq N+K-J+2 n-2 M, \text { or } J \geqq n-M
$$

which is a contradiction.

If $N+K-J<0$, then we can write

$$
N+K+J \geqq J-N-K+2 n-2 M, \text { or } N+K \geqq n-M,
$$

which implies $J \geqq n-M$ and so another contradiction. The proof of our lemma is now completed.

Let us now return to the proof of Theorem 1. Suppose $r^{*}(z)$ is a function meromorphic in $D$, continuous in $\bar{D}$ with $n-1$ or fewer poles in $D$ and such that $\left\|f-r^{*}\right\|_{U} \leqq\|f-r\|_{U}$. Some of the poles of $r^{*}$ may coincide with those of $f$ so let us relabel the $a_{k}$ 's so that $r^{*}$ has a pole (or poles) located at $a_{n-M+1}, \cdots, a_{n}$ but not at $a_{1}, a_{2}, \cdots, a_{n-M}$. The function

$$
R^{*}(z)=\left[f(z)-r^{*}(z)\right] \prod_{k=1}^{n}\left(\frac{z-a_{k}}{1-\bar{a}_{k} z}\right),
$$

will then be meromorphic in $D$, continuous in $\bar{D}$ and have $n-M-1$ or fewer poles in $D$. Furthermore $R^{*}\left(a_{k}\right)=B_{k}$ for $k=1,2, \cdots, n-M$, and $\left\|R^{*}\right\|_{U} \leqq\|R\|_{U}$. Consequently, as a result of Lemma $1, M=0, R^{*} \equiv R$ and $r^{*} \equiv r$.

Remarks. As mentioned before, it is possible to state Theorem 1 in a more general form by replacing $U$ by an arbitrary closed Jordan curve $\Gamma$ and by dropping the condition that the $a_{k}$ 's be distinct. Since the result in [1] that we previously referred to is given in that general setting, we refer to that paper for the details of this generalization.

We might finally remark that the range of results given here is quite narrow and that there are still many open questions. In particular, the problem of finding the meromorphic function having $k$ poles, where $k<n-1$, of best uniform approximation to $f$ on $\Gamma$ is open and is, in fact, the stumbling block for the extension of these results to the approximation of wider classes of functions. 


\section{REFERENCES}

1. S. J. Poreda, On the convergence of best uniform deviations, Trans. Amer. Math. Soc. 179 (1973), 49-59.

2. - A characterization of badly approximable functions, Trans. Amer. Math. Soc. 169 (1972), 249-256.

Department of Mathematics, Clark University, Worcester, Massachusetts 01610 